\title{
CONVEX RESOURCE ALLOCATION PROBLEMS ON DIRECTED ACYCLIC GRAPHS: DUALITY, COMPLEXITY, SPECIAL CASES, AND EXTENSIONS*
}

\author{
CLYDE L. MONMA, ALEXANDER SCHRIJVER, \\ MICHAEL J. TODD AND VICTOR K. WEI
}

\begin{abstract}
Consider the following resource allocation problem on a directed acyclic graph (the precedence graph). Each vertex has a known work load, and a fixed amount of total resource is available. The time required to process a vertex is inversely proportional to the amount of the resource allocated to it. The time to complete all of the work is the length of (time to complete) a longest chain in the graph. The problem of finding an allocation which minimizes the time required to complete all of the work subject to the limited resource availability can be formulated as a separable convex programming problem.

We use results from Lagrangian duality for convex programs, the length-width inequality, and Dilworth's Theorem for directed acyclic graphs, to obtain a strong relationship between optimal solutions of this problem and its dual. This allows us to obtain closed-form solutions for certain special classes of graphs, and leads to a generalization of the LYM Property for partially ordered sets. The computational complexity of the general problem is an open question. However, the ellipsoid method yields a fully-polynomial approximation scheme, and some light can be shed on the associated decision problem. The results of this paper are shown to extend to resource allocation problems on perfect graphs.
\end{abstract}

1. Introduction. Consider the following resource allocation problem. We are given a directed acyclic graph $G=(V, E)$ depicting the precedence relationship between the various jobs in a project, i.e., there is an edge from vertex $i$ to vertex $j$ if job $i$ must be completed before job $j$ can start. Each job $i$ has a weight (workload) $w_{i}>0$. The time it takes to complete job $i$ is equal to $\left(w_{i} / r_{i}\right)^{1 / k}$, where $r_{i}$ is the budget allocated to job $i$ and $k>0$ is a constant. A fixed amount of budget $B>0$ is appropriated for the entire project. The resource allocation problem is how to optimally allocate the budget among the jobs in order to complete the project in the least amount of time.

This model incorporates several important applications in resource allocation with precedence constraints and non-reusable resources. For $k=1$, the time to complete an individual job is inversely proportional to the money or manpower allocated. This corresponds to many actual government or industrial projects. For $k=2$, the application arises from VLSI (Very Large Scale Integration) circuit design. For an individual job, the product of the silicon area (resource) and the square of the time spent (time square) equals a constant (the workload). This area-time square relationship arises very often in VLSI designs, see, e.g. [UI].

*Received February 26, 1987; revised June 15, 1989.

AMS 1980 subject classification. Primary: 30C35. Secondary 90C25.

IAOR 1973 subject classification. Main: Programming/Network. Cross references: Allocation/Resources

OR/MS Index 1978 subject classification. Primary: 489 Networks/graphs. Secondary: 656 Programming/ Nonlinear/Convex.

Key words. Resource allocation, acyclic graphs, convex programming, Lagrangian duality, posets. 
Now we formulate our resource allocation problem as a separable convex programming problem. In a directed acyclic graph, a chain is a set of vertices in a path, and an antichain is a set of vertices no two of which are in a directed path. Note that two vertices in a chain are precedence constrained, while two in a antichain are not. Denote by $\mathbf{C}$ and $\mathbf{A}$ the set of all chains and antichains in the graph. Our resource allocation problem is, formally,

$\left(\mathrm{R}_{\mathrm{B}}\right) \quad \min t(\mathbf{r}) \equiv \max _{C \in \mathrm{C}} \sum_{i \in C}\left(w_{i} / r_{i}\right)^{1 / k}$

s.t.

$$
\begin{aligned}
& \sum_{i \in V} r_{i} \leqslant B, \\
& r_{i} \geqslant 0 \quad \text { for all } i \in V .
\end{aligned}
$$

In $\left(R_{B}\right)$ we can retain the standard weak inequalities $\mathbf{r} \geqslant 0$ by defining $t(\mathbf{r})$ as $+\infty$ if any $r_{i}$ is zero. Thus $t(\cdot)$ is an extended real-valued function. Similar comments apply to other problems stated below. In fact it is easy to obtain explicit positive lower bounds on the components of any optimal solution, and then the functions of concern will be finite and continuous (and usually differentiable) on their restricted domains.

A related problem is to minimize the total amount of resource used subject to an imposed completion time $T>0$, which can be expressed as

$$
\begin{array}{ll}
\min & b(\mathbf{s}) \equiv \sum_{i \in V} s_{i} \\
\text { s.t. } & \\
& \sum_{i \in C}\left(w_{i} / s_{i}\right)^{1 / k} \leqslant T \text { for all } C \in \mathbf{C}, \\
& s_{i} \geqslant 0 \quad \text { for all } i \in V .
\end{array}
$$

It is clear that an optimal solution to $\left(R_{B}\right)$ for any $B>0$ can be obtained from one to $\left(S_{T}\right)$ for any $T>0$ and vice versa; this relationship will be made precise in Lemma 1 . We will frequently be concerned with another equivalent problem, stated as $(P)$ below, which avoids the nondifferentiable objective function of $\left(R_{B}\right)$ and the nonlinear constraints of $\left(S_{T}\right)$. Denote by $x_{i}$ the normalized time $\left(w_{i} / s_{i}\right)^{1 / k} T^{-1}$ spent on vertex $i$. Then our problem is

$$
\min f(\mathbf{x}) \equiv \sum_{i \in V} w_{i} x_{i}^{-k}
$$

s.t.

$$
\begin{aligned}
& \sum_{i \in C} x_{i} \leqslant 1 \quad \text { for all } C \in \mathbf{C}, \\
& x_{i} \geqslant 0 \quad \text { for all } i \in V .
\end{aligned}
$$


The relationship between these problems is given by:

Lemma 1 (Equivalence Lemma). Let $T>0$ and $B>0$. Then $\left(R_{B}\right),\left(S_{T}\right)$ and $(P)$ all have unique optimal solutions, say $\mathbf{r}^{*}, \mathbf{s}^{*}$ and $\mathbf{x}^{*}$, and they are related by

$$
x_{i}^{*}=\left(w_{i} / s_{i}^{*}\right)^{1 / k} T^{-1}=\left(w_{i} / r_{i}^{*}\right)^{1 / k}\left(t\left(\mathbf{r}^{*}\right)\right)^{-1} .
$$

Moreover, $\max _{C \in \mathrm{C}} \sum_{i \in C} x_{i}^{*}=1$.

Proof. Let us show that $(P)$ has an optimal solution. Clearly, $\hat{\mathbf{x}}$ given by $\hat{x}_{i}=1 / V \mid$ for each $i \in V$ is feasible, with $f(\hat{\mathbf{x}})=|V|^{k} \sum_{i \in V} w_{i}$. Since each component in $f$ is nonnegative, any optimal solution must have each component no less than $\delta=\left[\left(\min _{i \in V} w_{i}\right) / f(\hat{\mathbf{x}})\right]^{-1 / k}>0$. Hence $(P)$ can be restricted to those feasible $\mathbf{x}$ with $\delta \leqslant x_{i} \leqslant 1$ for all $i \in V$, giving a compact set on which $f$ is finite, continuous and strictly convex. It follows that $(P)$ has a unique optimal solution $\mathbf{x}^{*}$.

Existence of optimal solutions to $\left(R_{B}\right)$ and $\left(S_{T}\right)$ follows similarly, and it is easy to show that if $\mathbf{x}^{*}, \mathbf{s}^{*}$ and $\mathbf{r}^{*}$ are related as shown, then $\mathbf{s}^{*}$ and $\mathbf{r}^{*}$ are optimal in their respected problems, and vice versa. Since $\mathbf{x}^{*}$ is unique, this establishes that $\mathbf{r}^{*}$ and $\mathbf{s}^{*}$ are unique also.

The final claim is trivial; if it were false, we could scale up $\mathrm{x}^{*}$, retain feasibility, and reduce $f$.

We note that the number of constraints in $(P)$ can grow exponentially fast as a function of the size of $G$. However, these constraints can be represented compactly, since for any particular $\overline{\mathbf{x}}$, we can test feasibility to $(P)$ in polynomial time by solving a longest chain problem in the directed acyclic graph $G$ with weight $\bar{x}_{i}$ for each vertex $i$, and checking to see that the length of the longest chain does not exceed one. If $\overline{\mathbf{x}}$ is infeasible for $(P)$, this approach solves the "separation" problem by providing a chain $\bar{C}$ whose corresponding constraint is violated.

In $\$ 2$, we use results from Lagrangian duality for convex programming (see e.g. [Ro1]), the length-width inequality [Le] and Dilworth's Theorem [Di] for directed acyclic graphs to obtain a strong relationship between $(P)$ and its dual problem. This result can also be obtained using an equivalent pair of dual problems and results from convex network optimization; in the notation of Rockafellar [Ro2], these dual problems are, respectively, optimal differential and optimal distribution problems. These problems were suggested by reformulations due to the associate editor and one of the referees. However, while the new primal has only $O(|E|)$ (dual network flow) constraints and the new dual has only $O(|V|)$ (network flow) constraints, we prefer the original problems for their application to specific graphs in $\$ 5$, and their generalizations to cliques and anti-cliques in perfect graphs in $\$ 6$. General duality is necessary in this last case. $\$ 2$ also states results for an alternative symmetric duality for problem $\left(R_{B}\right)$.

In practice, one would probably solve $\left(R_{B}\right)$ by solving the convex network optimization problems. Here, however, we are more interested in their theoretical computational complexity, which remains open. (See [GJ] for an excellent introduction to the field of computational complexity.)

In $\S 3$, the ellipsoid method [BGT, GLS2] is used to obtain a fully-polynomial approximation scheme for $(P)$. In $\S 4$, some insight is presented for the decision problem associated with $(P)$; namely "Given an input value $F$ and an instance of $(P)$, does the optimal objective value of $(P)$ exceed $F$ ?"

In $\$ 5$, we obtain closed-form solutions for certain special directed acyclic graphs such as serial-parallel graphs and some level graphs; this also leads to a generalization of the LYM Property for partially ordered sets. In $\$ 6$, we show how to extend our results to resource allocation problems on perfect graphs. 
2. Strong duality. In this section we prove a strong duality result between $(P)$ and the problem

$$
\max _{\mathbf{y} \geqslant \mathbf{0}} g(\mathbf{y}) \equiv\left\{\sum_{i \in V}(k+1)\left(\frac{w_{i}}{k^{k}}\right)^{1 /(k+1)} y_{i}^{k /(k+1)}-\max _{A \in \mathbf{A}} \sum_{i \in A} y_{i}\right\}
$$

(Recall that $\mathbf{A}$ is the collection of all antichains.) While we will use general methods, it is also possible to use duality for convex network optimization as in [Ro2] to establish this result. Let us state the corresponding problems.

Let $\hat{G}=(\hat{V}, \hat{E})$ be obtained from $G$ as follows. Its vertex set $\hat{V}$ consists of the copies, $i^{\prime}$ and $i^{\prime \prime}$, of each vertex $i$ of $G$, together with an initial vertex $p$ and a terminal vertex $q$. Its edge set $\hat{E}$ is the union of $\hat{E}_{V} \equiv\left\{\left(i^{\prime}, i^{\prime \prime}\right): i \in V\right\}, \hat{E}_{E} \equiv\left\{\left(i^{\prime \prime}, j^{\prime}\right)\right.$ : $(i, j) \in E\} \cup\left\{\left(p, i^{\prime}\right): i \in V\right.$ has indegree 0$\} \cup\left\{\left(j^{\prime \prime}, q\right): j \in V\right.$ has outdegree 0$\}$, and $(q, p)$. Thus vertex $p$ represents the start of the process, $i^{\prime}$ the start and $i^{\prime \prime}$ the end of the work at vertex $i$, and $q$ the finishing of all the work. Let $u_{\hat{i}}$ denote the time at which vertex $\hat{i} \in \hat{V}$ is reached. Then it is clear that $(P)$ is equivalent to

$$
\begin{aligned}
& \min \sum_{i \in V} w_{i}\left(u_{i^{\prime \prime}}-u_{i^{\prime}}\right)^{-k} \\
& u_{j}-u_{i} \geqslant 0 \quad \text { for all }(\hat{i}, \hat{j}) \in \hat{E}_{V} \cup \hat{E}_{E}, \\
& u_{p}-u_{q}=-1 .
\end{aligned}
$$

This is an optimal differential problem, and it is dual to the optimal distribution problem:

$$
\begin{aligned}
& \max \sum_{i \in V}(k+1)\left(\frac{w_{i}}{k^{k}}\right)^{1 /(k+1)} \nu_{i^{\prime} i^{\prime \prime}}^{k /(k+1)}-\nu_{q p} \\
& \sum_{\hat{h}:(\hat{h}, \hat{i}) \in \hat{E}} \nu_{\hat{h} \hat{i}}-\sum_{\hat{j}:(\hat{i}, \hat{j}) \in \hat{E}} \nu_{i \hat{j}}=0 \text { for all } \hat{i} \in \hat{V}, \\
& \mathbf{v} \geqslant \mathbf{0} .
\end{aligned}
$$

We will see below that $\left(D^{\prime}\right)$ is equivalent to $(D)$.

THEOREM 2 (Strong Duality Theorem). For any $\mathbf{x}$ feasible for $(P)$ and any $\mathbf{y} \geqslant 0$, $f(\mathbf{x}) \geqslant g(\mathbf{y})$. Furthermore, there exist unique optimal solutions $\mathbf{x}^{*}$ and $\mathbf{y}^{*}$ to $(P)$ and $(D)$, respectively, which satisfy $f\left(\mathbf{x}^{*}\right)=g\left(\mathbf{y}^{*}\right)$ and $y_{i}^{*}=k w_{i}\left(x_{i}^{*}\right)^{-k-1}$ for all $i \in V$.

Proof. The Lagrangian dual of $(P)$ is

$$
\max _{\boldsymbol{\mu} \geqslant \mathbf{0}} h(\boldsymbol{\mu}) \equiv\left\{\min _{\mathbf{x} \geqslant \mathbf{0}} \sum_{i \in V}\left(w_{i} x_{i}^{-k}+\left(\sum_{C: i \in \mathrm{C}} \mu_{C}\right) x_{i}\right\}-\sum_{C \in \mathrm{C}} \mu_{C}\right\}
$$

It is well known that $f(\mathbf{x}) \geqslant h(\mu)$ for any $\mathbf{x}$ feasible in $(P)$ and any $\boldsymbol{\mu} \geqslant 0$; moreover, since $(P)$ has a unique optimal solution $\mathbf{x}^{*}$ at which all functions in $(P)$ are differentiable, and $(P)$ has linear constraints, $(L)$ has an optimal solution $\mu^{*}$ and $f\left(\mathbf{x}^{*}\right)=h\left(\mu^{*}\right)$ (see for example [Ro1]). It therefore remains to relate $(L)$ to $(D)$ and prove the uniqueness of $y^{*}$ and its relationship to $\mathbf{x}^{*}$. 
Now the minimand in $h(\mu)$ is convex and separable in $\mathbf{x}$. By setting its partial derivative with respect to $x_{i}$ equal to zero we find

$$
\begin{aligned}
& \max _{\boldsymbol{\mu} \geqslant \mathbf{0}} h(\boldsymbol{\mu})=\max _{\boldsymbol{\mu} \geqslant \mathbf{0}}\left\{\sum_{i \in l}(k+1)\left(\frac{w_{i}}{k^{k}}\right)^{1 /(k+1)}\left(\sum_{C: i \in C} \mu_{C}\right)^{k /(k+1)}-\sum_{C \in \mathbf{C}} \mu_{C}\right\} \\
& =\max _{\substack{\mu \geqslant 0 \\
\mathbf{y} \geqslant \mathbf{0} \\
y_{i} \leqslant \sum_{C: i \in C} \mu_{c}}} \mu_{c}\left\{\sum_{i \in V^{\prime}}(k+1)\left(\frac{w_{i}}{k^{k}}\right)^{1 /(k+1)}\left(y_{i}\right)^{k /(k+1)}-\sum_{C \in \mathbf{C}} \mu_{C}\right\} \\
& =\max _{\mathbf{y} \geqslant \mathbf{0}} g(\mathbf{y}) \text {. }
\end{aligned}
$$

The second equality follows since for any fixed value of $\mu$, each summand can be maximized separately by setting $y_{i}=\sum_{C: i \in C} \mu_{C}$. The final equality follows from a weighted version of Dilworth's Theorem [Di] which states that the minimum cover of weighted vertices by chains equals the maximum-weight antichain. This proves that $(P)$ and $(D)$ satisfy the strong duality relationship since $(P)$ and $(L)$ do.

To prove the final claim, we need to use the length-width inequality [Le] for directed acyclic graphs. Let $\mathbf{x}$ be a positive feasible solution to $(P)$, and let $\mathbf{y}$ be a feasible solution to $(D)$. Then

$$
\max _{\mathcal{A} \in \mathbf{A}} \sum_{i \in A} y_{i} \geqslant\left(\max _{C \in \mathbf{C}} \sum_{i \in C} x_{i}\right)\left(\max _{A \in \mathbf{A}} \sum_{i \in A} y_{i}\right) \geqslant \sum_{i \in V} x_{i} y_{i} .
$$

To bound the right-hand side, we consider the (generalized) arithmetic-geometric mean inequality, which states that, if $\alpha$ and $\beta$ are positive with $\alpha+\beta=1$, and $c$ and $d$ are nonnegative, then $\alpha c+\beta d \geqslant c^{\alpha} d^{\beta}$, with equality if and only if $c=d$. Applying this result with $\alpha=k /(k+1), \beta=1 /(k+1), c=c_{i}=x_{i} y_{i} / k$ and $d=d_{i}=w_{i} x_{i}^{-k}$, we find that

$$
x_{i} y_{i} \geqslant-w_{i} x_{i}^{-k}+(k+1)\left(\frac{w_{i}}{k^{k}}\right)^{1 /(k+1)} y_{i}^{k /(k+1)} .
$$

Thus, from (2.2) and (2.3) we find that

$$
f(\mathbf{x}) \geqslant g(\mathbf{y})
$$

which gives an alternative proof of weak duality; moreover, equality holds in (2.4) only if equality holds in (2.3) for all $i$, which implies that $c_{i}=d_{i}$, and hence, $y_{i}=k w_{i} x_{i}^{-k-1}$ for all $i \in V$.

This result can also be obtained from the monotropic network duality of Rockafellar [Ro2]. Clearly, $(P)$ and $\left(P^{\prime}\right)$ are equivalent (with $x_{i}$ corresponding to the "potential difference" $\left.u_{i}^{\prime \prime}-u_{i}^{\prime}\right)$. Next, $\left(P^{\prime}\right)$ and $\left(D^{\prime}\right)$ are dual-here both problems have reversed objective functions, and the flow $\mathbf{v}$ is the negative of the flow that results from network duality. Finally, if we decompose the flow $\mathbf{v}$ into weighted chains from $p$ to $q$ (together with flow on the return edge $(q, p)$ ), we get a problem on the enlarged graph $\hat{G}$ that is equivalent to the problem in (2.1) on the original graph $G$. As we have seen above, this is equivalent to $(D)$. Note that $y_{i}$ corresponds to $\sum_{C: i \in C} \mu_{c}$ and hence to $\nu_{i^{\prime} i^{\prime \prime}}$; then the Network Equilibrium Theorem of Chapter 8 of [Ro2] establishes the relationship between $\mathbf{x}^{*}$ and $\mathbf{y}^{*}$. 
To conclude this section, we state an alternative symmetric duality for the problem $\left(R_{B}\right)$ :

$$
\min t(\mathbf{r}) \equiv \max _{C \in \mathbf{C}} \sum_{i \in C}\left(w_{i} / r_{i}\right)^{1 / k}
$$

$\left(\mathrm{R}_{\mathrm{B}}\right)$

$$
\sum_{i \in V} r_{i} \leqslant B
$$

$$
\mathbf{r} \geqslant \mathbf{0} .
$$

Consider the problem

$\left(\mathrm{DR}_{\mathrm{B}}\right)$

$$
\begin{aligned}
\min u(\mathbf{z}) & \equiv \max _{A \in \mathbf{A}} \sum_{i \in A}\left(z_{i}^{k+1} / w_{i}\right)^{1 / k}, \\
\sum_{i \in V} z_{i} & \geqslant B, \\
\mathbf{z} & \geqslant \mathbf{0} .
\end{aligned}
$$

Theorem 3 (Symmetric Duality). (a) Weak Duality: Let $\overline{\mathbf{r}}$ and $\overline{\mathbf{z}}$ be feasible solutions to $\left(R_{B}\right)$ and $\left(D R_{B}\right)$, respectively, then $t(\overline{\mathbf{r}}) \cdot u(\overline{\mathbf{z}}) \geqslant B$.

(b) Strong Duality: Both $\left(R_{B}\right)$ and $\left(D R_{B}\right)$ have unique optimal solutions $\hat{\mathbf{r}}$ and $\hat{\mathbf{z}}$, respectively, with $\hat{\mathbf{r}}=\hat{\mathbf{z}}$. Furthermore, $t(\hat{\mathbf{r}}) \cdot u(\hat{\mathbf{z}})=B$.

A proof can be found in [MSTW].

3. Fully polynomial approximation scheme. In this section we show how the ellipsoid method (see e.g., [BGT] and [GLS2]) provides a fully-polynomial approximation scheme for $(P)$; more precisely, we can obtain a feasible solution with objective function value within any $\epsilon>0$ of the optimal value, in time polynomial in the length of the input and $\log (1 / \epsilon)$ for fixed $k$. (Similar arguments apply to $\left(R_{B}\right)$ if $k=1$.) We assume that each $w_{i}$ is a positive integer. The results of this section also follow from general arguments in [GLS2].

Let $|V|=n$, and let $\mathbf{e}$ be the vector with $e_{i}=1$ for all $i \in V$. Let $S^{\prime}$ denote the feasible region of $(P)$. Note that $\mathbf{e} / n \in S^{\prime}$ with objective function $f(\mathbf{e} / n)=w n^{k}$, where $w=\sum_{i \in V} w_{i}$. Thus, we can confine ourselves to points with $f(\mathbf{x}) \leqslant f(\mathrm{e} / n)$, which implies that $x_{i} \geqslant\left(w_{i} / w\right)^{1 / k} / n$ for all $i \in V$. Let $\underline{x}_{i}=\min \left\{\left(w_{i} / w\right)^{1 / k} / n, 1 / 3 n\right\}$, and let $S=\left\{x \in S^{\prime}: x_{i} \geqslant \underline{x}_{i}\right.$ for all $\left.i \in V\right\}$.

Then $S$ contains the optimal solution, and since $\{x:(1 / 3 n) e \leqslant x \leqslant(1 / n) e\} \subseteq S \subseteq$ $\{x: 0 \leqslant x \leqslant e\}$,

$$
B((2 / 3 n) \mathbf{e}, 1 / 3 n) \subseteq S \subseteq B(\mathbf{e} / 2, \sqrt{n} / 2),
$$

where $B(\mathbf{x}, r)$ denotes the Euclidean ball around $\mathbf{x}$ of radius $r$.

Our problem is to minimize $f(\mathbf{x})$ for $\mathbf{x} \in S$. The ellipsoid method generates a sequence $\left\{E_{j}\right\}$ of ellipsoids with centers $\mathbf{x}^{j}$, such that if $f_{j}=\min \left\{f\left(\mathbf{x}^{m}\right): 0 \leqslant m<j\right.$, $\left.\mathbf{x}^{m} \in S\right\}$ (this minimum has value $\infty$ if there is no such $\mathbf{x}^{m}$ ), then

$$
E_{j} \supseteq\left\{\mathbf{x} \in S: f(\mathbf{x}) \leqslant f_{j}\right\} .
$$

By setting $\mathbf{x}^{0}=\mathbf{e} / 2$ and $E_{0}=B(\mathbf{e} / 2, \sqrt{n} / 2)$, we see that (3.2) holds for $j=0$. Assuming that (3.2) holds for some $j \geqslant 0$, we show how to find $E_{j+1}$ so that (3.2) also 
holds for $j+1$, and so that volume $\left(E_{j+1}\right) \leqslant \exp (-1 / 4 n)$ volume $\left(E_{j}\right)$. We represent each ellipsoid by its center $\mathbf{x}^{j}$, and a symmetric, positive-definite matrix $A_{j}$, so that $E_{j}=\left\{\mathbf{x}:\left(\mathbf{x}-\mathbf{x}^{j}\right)^{T} A_{j}^{-1}\left(\mathbf{x}-\mathbf{x}^{j}\right) \leqslant 1\right\}$.

To determine $E_{j+1}$, we first check whether $\mathrm{x}^{j} \in S$. If $x_{i}^{j}<\underline{x}_{i}$ for some $i \in V$, we set $a_{i}=-1$ and $a_{h}=0$ for all $h \neq i$, and note that

$$
S \subseteq\left\{\mathbf{x}: \mathbf{a}^{T} \mathbf{x} \leqslant \mathbf{a}^{T} \mathbf{x}^{j}\right\}
$$

If $\mathbf{x}^{j} \geqslant \underline{\mathbf{x}}$, we find a longest chain $C$ in $G$ with the weight $x_{i}^{j}$ on each vertex $i \in V$. If the length of $C$ is greater than 1 , set $a_{i}=1$ if $i \in C$, and set $a_{i}=0$ otherwise; then (3.3) again holds.

Now suppose $\mathbf{x}^{j} \in S$. We then set $\mathbf{a}=\nabla f\left(\mathbf{x}^{j}\right)$, so that $a_{i}=-k w_{i}\left(x_{i}^{j}\right)^{-k-1}$ for all $i \in V$. Note that, since $\underline{\mathbf{x}}^{j} \leqslant \mathbf{x}^{j} \leqslant \mathbf{1}$, we can bound the components of $\mathbf{a}$ by

$$
k w_{i} \leqslant\left|a_{i}\right| \leqslant k w_{i} n^{k+1} \max \left\{3^{k+1},\left(w / w_{i}\right)^{(k+1) / k}\right\},
$$

for all $i \in V$. If $f(\mathbf{x}) \leqslant f\left(\mathbf{x}^{j}\right)$, we have $\mathbf{a}^{T} \mathbf{x} \leqslant \mathbf{a}^{T} \mathbf{x}^{j}$.

Using this observation if $\mathbf{x}^{j}$ is feasible, or using (3.3) if it is not, together with (3.2), we find that

$$
\left\{\mathbf{x} \in E_{j}: \mathbf{a}^{T} \mathbf{x} \leqslant \mathbf{a}^{T} \mathbf{x}^{j}\right\} \supseteq\left\{\mathbf{x} \in S: f(\mathbf{x}) \leqslant f_{j+1}\right\} .
$$

Hence, (3.2) will hold for $j+1$ as long as $E_{j+1}$ contains the semiellipsoid on the left side of (3.5). The minimum volume such ellipsoid $\bar{E}_{j+1}$ is given by its center $\overline{\mathbf{x}}^{j+1}$ and the symmetric positive definite matrix $\overline{\mathbf{A}}_{j+1}$ defined by

$$
\begin{aligned}
& \overline{\mathbf{x}}^{j+1}=\mathbf{x}^{j}-\tau A_{j} \mathbf{a} /\left(\mathbf{a}^{T} A_{j} \mathbf{a}\right)^{1 / 2}, \\
& \bar{A}_{j+1}=\delta\left(A_{j}-\sigma A_{j} \mathbf{a}^{T} A_{j} / \mathbf{a}^{T} A_{j} \mathbf{a}\right),
\end{aligned}
$$

where $\tau=1 /(n+1), \sigma=2 /(n+1)$, and $\delta=n^{2} /\left(n^{2}-1\right)$ (see, e.g., [BGT]). Then volume $\left(\bar{E}_{j+1}\right) \leqslant \exp (-1 / 2(n+1)) \cdot \operatorname{volume}\left(E_{j}\right)$. Grotschel, Lovasz and Schrijver [GLS1] show that, if $\delta$ is instead chosen as $2 n^{2} /\left(2 n^{2}-3\right)$, then $\overline{\mathbf{x}}^{j+1}$ and $\overline{A_{j+1}}$ can be rounded to $\mathbf{x}^{j+1}$ and $A_{j+1}$ using a number of digits after the decimal point that is polynomial in $j$ and $n$, and the resulting ellipsoid $E_{j+1}$ will still satisfy (3.2) for $j+1$, and volume $\left(E_{j+1}\right) \leqslant \exp (-1 / 4 n)$ volume $\left(E_{j}\right)$.

We want to find a point $\overline{\mathbf{x}} \in S$ with

$$
f(\overline{\mathbf{x}}) \leqslant \min \{f(\mathbf{x}): \mathbf{x} \in S\}+\epsilon
$$

in time polynomial in $n, \log w$ and $\log (1 / \epsilon)$. It suffices to show that some $\mathbf{x}^{j}$ satisfies this inequality for a polynomially-bounded $j$. Let $\mathbf{x}^{*}$ be the optimal solution to $(P)$. Let $\gamma=\min \left\{(3 n)^{-k}(\epsilon / w), 1\right\}$. Note that $f\left(\mathbf{x}^{*}\right) \geqslant w$ and that $f(\mathbf{x}) \leqslant(3 n)^{k} w$ for all $\mathbf{x} \in B_{1}=B((2 / 3 n) \mathbf{e}, 1 / 3 n)$. Consider $B_{\gamma}=\left\{\gamma \mathbf{x}+(1-\gamma) \mathbf{x}^{*}: \mathbf{x} \in B_{1}\right\} \subseteq S$. By convexity, for any $\mathbf{z}=\gamma \mathbf{x}+(1-\gamma) \mathbf{x}^{*} \in B_{\gamma}$ where $x \in B_{1}$, we have $f(\mathbf{z}) \leqslant \gamma f(\mathbf{x})+(1-$ $\gamma) f\left(\mathbf{x}^{*}\right)<f\left(\mathbf{x}^{*}\right)+\gamma f(\mathbf{x}) \leqslant f\left(\mathbf{x}^{*}\right)+\epsilon$. Hence, any point in $B_{\gamma}$ solves our problem. Now $B_{\gamma}$ has volume $\gamma^{n}(2 /(3 n \sqrt{n}))^{n}$ times that of $E_{0}$. Thus, in $4 n^{2}(\log (1 / \gamma)+$ $\log (3 n \sqrt{n} / 2))$ steps, $E_{j}$ will have volume smaller than that of $B_{\gamma}$. By (3.2), this implies that some $\mathbf{x}^{m}, 0 \leqslant m<j$, is feasible and has $f\left(\mathbf{x}^{m}\right) \leqslant f\left(\mathbf{x}^{*}\right)+\epsilon$. We have therefore shown that the ellipsoid method gives a fully-polynomial approximation scheme for $(P)$. The case for $\left(R_{B}\right)$ is similar, noting that a subgradient of $t(\mathbf{r})$ is available by 
finding a longest chain with weights $\left(w_{i} / r_{i}\right)^{1 / k}$, and then using the gradient of this term in $t(\mathbf{r})$. (We need $k=1$ so that $\underline{\mathbf{x}}$ is rational.)

4. The associated decision problem. The computational complexity of problem $(P)$ and its associated decision problem remain open questions. We conjecture that these problems are solvable in polynomial time. In this section, we present an approach which might eventually verify this conjecture.

Let $A$ be an integral $m \times n$ matrix and let $\mathbf{b}$ be an integer $m$-vector. Consider the following convex programming problem $\left(P^{*}\right)$ :

$$
\begin{aligned}
\operatorname{minimize} & \sum_{i=1}^{n} \frac{1}{x_{i}} \\
\text { subject to } A \mathbf{x} & =\mathbf{b}, \\
\mathbf{x} & >0 .
\end{aligned}
$$

It is not difficult to see, using Lagrangian theory, that the unique optimal solution $\mathbf{x}^{*}$ to $\left(P^{*}\right)$ consists of algebraic numbers. In fact, it is the first part of the unique positive solution $\left(\mathbf{x}^{*}, \mathbf{y}^{*}\right)$ to the following system of algebraic equations $\left(E^{*}\right)$ in variables $(\mathbf{x}, \mathbf{y})$ :

$$
\begin{aligned}
A \mathbf{x} & =\mathbf{b}, \\
\frac{1}{x_{i}^{2}} & =(\mathbf{y} A)_{i} \quad 1 \leqslant i \leqslant n .
\end{aligned}
$$

This relates as follows to our problem $(P)$, restricting ourselves to the case $w_{i}=1$ for all $i$, and $k=1$. It is clear that the optimal solution to the convex programming problem $(P)$ satisfies at least one chain constraint at equality and has all positive components. Hence it is also a solution to a problem of the form $\left(P^{*}\right)$, where $A x=b$ includes all tight chain constraints.

It would now be very helpful if we could find a polynomial upper bound on the degree and in the size of the height (i.e., the log of the maximum absolute value of the coefficients) of the minimal polynomials of the $x_{i}^{*}$. Then, with the fully polynomial approximation scheme of Section 4, we can apply the following theorem of Lovász [Lo3, p. 38, Theorem 1.4.7]:

Given a rational number $q$, an integer $h$, and the information that there exists a real algebraic number $\alpha$ whose minimal polynomial has degree and height-size at most $h$ and with $|q-\alpha|<2^{-4 h^{3}}$, we can compute the minimal polynomial of $\alpha$ in time polynomially bounded by $h$ and $\langle q\rangle$, where $\langle q\rangle$ is the number of bits necessary to specify $q$.

Denote by $\alpha$ the optimal objective function value for $(P)$, denoted by $p(x)$ the minimal polynomial of $\alpha$, and let $h$ be an integer at least the degree and height-size of $p(x)$.

The decision problem is: "Given a rational $q$, is $q \leqslant \alpha$ ?". One line of attacking this question is as follows. First apply the fully-polynomial approximation scheme of $\S 4$ to obtain an interval $\left(q_{1}, q_{2}\right)$ which contains $\alpha$ with $q_{1}$ and $q_{2}$ rational, and $\left|q_{2}-q_{1}\right|<$ $2^{-4 h^{3}}$. If $q \leqslant q_{1}$ or $q \geqslant q_{2}$ then we are done. If not, use Lovasz' Theorem stated above to compute the minimum polynomial $p(x)$ of $\alpha$. Since $\alpha$ is the only zero of $p(x)$ in $\left(q_{1}, q_{2}\right)$ checking the sign of $p(q)$ will tell us whether $q \leqslant \alpha$ or not.

This approach would require polynomial time if we could find a polynomial upper bound on the degree and height-size of the coefficients of the unique positive solutions of $\left(E^{*}\right)$. We leave this as an open problem.

5. Closed-form solution for special graphs. In this section, we present closedform solutions to our resource allocation problems on two special classes of directed 
acyclic graphs: series-parallel graphs and certain level graphs. We solve the former by the equivalent load method, and the latter by coalescing certain vertices. Our analysis also leads to a generalization of the LYM Property for partially ordered sets.

5.1. Series-parallel graphs. Let $G_{1}$ and $G_{2}$ be two directed acyclic graphs. The series connection of $G_{1}$ and $G_{2}$, denoted by $G_{1} \rightarrow G_{2}$, is obtained by taking the union of $G_{1}$ and $G_{2}$ and adding an edge from each vertex of $G_{1}$ with outdegree zero to every vertex in $G_{2}$ with indegree zero. The parallel connection of $G_{1}$ and $G_{2}$, denoted by $G_{1} \| G_{2}$, is the union of $G_{1}$ and $G_{2}$. A series-parallel graph is defined recursively as a directed acyclic graph with a single vertex, or one which can be constructed by the series connection or the parallel connection of two series-parallel graphs.

A closed-form solution for the resource allocation problem on a series-parallel graph can be obtained by the method of "equivalent load." Given an instance of $\left(R_{B}\right)$, the equicalent load of the graph is defined to be the product of the total budget and the $k$ th power of the minimum total completion time. This product is a constant, as follows easily from the Equivalence Lemma.

THEOREM 4. Let $w_{1}$ and $w_{2}$ be the equivalent loads of two series-parallel graphs $G_{1}$ and $G_{2}$. The equilalent load of the parallel connection $G=G_{1} \| G_{2}$ is $w_{1}+w_{2}$; the equivalent load of the series connection $G=G_{1} \rightarrow G_{2}$ is $\left(w_{1}^{1 /(k+1)}+w_{2}^{1 /(k+1)}\right)^{k+1}$.

Proof. Let $B_{j}$ be the total budget allocated to the vertices of $G_{j}$ for $j=1,2$, where $B_{1}+B_{2}=B$ is the budget for $G$. Let $w_{j}$ be the equivalent work loads for $G_{j}$ for $j=1,2$. The minimum completion time is $T_{j}=\left(w_{j} / B_{j}\right)^{1 / k}$ for $j=1,2$. Let $T$ be the minimum completion time for $G$. In the parallel connection, we have

$$
T=\min _{B_{1}+B_{2}=B} \max \left\{T_{1}, T_{2}\right\}=\min _{B_{1}+B_{2}=B}\left\{\left(\frac{w_{1}}{B_{1}}\right)^{1 / k},\left(\frac{w_{2}}{B_{2}}\right)^{1 / k}\right\}=\left(\frac{w_{1}+w_{2}}{B}\right)^{1 / k} .
$$

Therefore, the equivalent load is $w_{1}+w_{2}$. In the series connection

$$
\begin{aligned}
T & =\min _{B_{1}+B_{2}=B}\left(T_{1}+T_{2}\right)=\min _{B_{1}+B_{2}=B}\left(\frac{w_{1}}{B_{1}}\right)^{1 / k}+\left(\frac{w_{2}}{B_{2}}\right)^{1 / k} \\
& =B^{-1 / k}\left(w_{1}^{1 /(k+1)}+w_{2}^{1 /(k+1)}\right)^{(k+1) / k} .
\end{aligned}
$$

Therefore, the equivalent load is $\left(w_{1}^{1 /(k+1)}+w_{2}^{1 /(k+1)}\right)^{k+1}$.

Note that the optimum budget partition for the parallel connection yields

$$
B_{j}=\frac{w_{j}}{w_{1}+w_{2}} B \text { for } j=1,2 \text {, and } T_{1}=T_{2} .
$$

The optimum budget partition for the series connection yields

$$
B_{j}=\frac{w_{j}^{1 /(k+1)}}{w_{1}^{1 /(k+1)}+w_{2}^{1 /(k+1)}} B, \quad \text { and } \quad T_{j}=\frac{w_{j}^{1 /(k+1)}}{w_{1}^{1 /(k+1)}+w_{2}^{1 /(k+1)}} T, \quad \text { for } j=1,2 .
$$

The minimum completion time of a series-parallel graph $G$ is $T=\left(w_{G} / B\right)^{1 / k}$, where $w_{G}$ can be obtained by recursive application of the above theorem. The optimum budget assignment can be obtained easily from Lemma 1.

5.2. Level graphs. Closed-form solutions can be obtained for a class of graphs whose vertices can be partitioned into antichains satisfying certain conditions. Roughly speaking, we treat such a graph as if it were a series-parallel graph with the vertices in each antichain being in parallel, and with the antichains being in series. We coalesce the vertices in each antichain in the partition into a single vertex. The 
resulting graph consists of a single chain, which is solved by the series-parallel method of $\$ 5.1$. The dual of $\S 2$ is used to verify the solution. The conditions under which this method yields the correct solution are presented in this section. These conditions turn out to be an interesting generalization of the LYM property of partially ordered sets.

In a directed acyclic graph $G$, a vertex $i$ is a predecessor of a vertex $j$ if there is a directed path from $i$ to $j$; a vertex $i$ is an immediate predecessor of $j$ if there is no other vertex $k$ on any directed path from $i$ to $j$. We say that $i$ and $j$ are comparable if there is a chain containing both $i$ and $j$. A level function is a mapping $l$ from the vertices of $G$ to the integers, such that $l(i)=0$ for every vertex $i$ with indegree zero, and $l(j)=l(i)+1$ whenever vertex $i$ is an immediate predecessor of vertex $j$. A graph with such a function on its vertices is called a level graph. The vertices mapped to any integer $r$ form an antichain $L_{r}$, called the $r$ th level.

Under certain conditions, the vertices on each level can be coalesced into a single vertex whose work load is the sum of the loads for all of the vertices in that level. The resulting graph consists of vertices in series, and a closed-form solution can be obtained using the method of $\S 6.1$.

For any $X \subseteq V$, denote by $\|X\|$ the sum $\sum_{i \in X} w_{i}$. If the coalescing method applies, then the equivalent load of the entire graph is $w_{G}=\left(\sum_{r}\left\|L_{r}\right\|^{1 /(k+1)}\right)^{k+1}$. Given a total budget $B$, the total completion time is $T=\left(w_{G} / B\right)^{1 / k}$. The budget allocated to level $L_{r}$ is $B_{r} \equiv\left(\left\|L_{r}\right\| / w_{G}\right)^{1 /(k+1)} B$, and the budget allocated to each vertex $i$ on level $L_{r}$ is $b_{i}=w_{i}\left\|L_{r}\right\|^{-1} B_{r}$. All jobs on the same level take the same amount of time. On level $L_{r}$, each job takes time $T_{r}=\left(\left\|L_{r}\right\| / w_{G}\right)^{1 /(k+1)} T$. We can verify that $w_{i}=b_{i} T_{r}^{k}$ when $i \in L_{r}$.

Whether the coalescing method gives the correct solution depends upon whether the graph satisfies the LYM property. The LYM property, due independently to Lubell, Yamamoto, and Meschalkin, is defined for partially ordered sets with rank functions which are equivalent to level graphs; see Greene and Kleitman [GK] for an excellent exposition. It is concerned with the weight of maximum antichains, "regular" chain coverings, and certain normalized matching conditions. Graphs satisfying the LYM property include the planar grid, the hypercube, certain bounded integer lattices, and many others. On these graphs, the coalescing method can be used to obtain closed-form solutions for the convex programming problem. The LYM property is usually described in terms of three equivalent conditions. Our convex programming perspective adds a fourth equivalent condition to the collection. Let $G$ be a level graph with all weights equal to one. A regular chain covering is a collection of chains (with multiplicities allowed), such that every chain intersects every level $L_{r}$ and the number of chains passing through a vertex depends only on the level. For $X \subseteq V$, we let $\Gamma(X)$ denote the set of vertices comparable to some vertex in $X$. By convention, $X \subseteq \Gamma(X)$.

THEOREM 5 (The LYM Property). Let $G$ be a level graph with $s$ levels and weights $w_{i}=1$. Let $\mathbf{y}$ denote the vector defined by $y_{i}=1 /\left\|L_{r}\right\|$, where $i \in L_{r}$. Let $w_{G}=$ $\left(\sum_{r}\left\|L_{r}\right\|^{1 /(k+1)}\right)^{k+1}$. Then the following four conditions are equivalent:

1. $\left(k w_{G}\right) \mathbf{y}$ is an optimal solution vector to the dual convex programming problem $(D)$.

2. $\sum_{i \in A} y_{i} \leqslant 1$ for all antichains $A \in \mathbf{A}$.

3. Each $L_{r}$ is a maximal antichain and there exists a regular chain covering.

4. $\sum_{i \in \Gamma^{\prime}(x) \cap L_{r+1}} y_{i} \geqslant \sum_{i \in X} y_{i}$ for any $X \subseteq L_{r}$ and any $L_{r}, 1 \leqslant r<s$.

The LYM property can be further generalized to graphs that can be partitioned into antichains. We provide a proof below for this more general case.

Let $G$ be a directed acyclic graph with node weights $\mathbf{w}$, and let $L_{1}, L_{2}, \ldots, L_{s}$ be a partition of the vertices of $G$ into $s$ antichains. Since there may be nonuniform vertex 
weights, we refine the definition of regular chain covering as follows. A weighted regular chain covering is a collection of chains (with perhaps nonintegral multiplicities) such that every chain intersects every $L_{r}$, and the ratio of the total multiplicities of the chains passing through any two vertices in the same $L_{i}$ equals the ratio of their weights. In other words, there are constants $\alpha_{1}, \alpha_{2}, \ldots, \alpha_{s}$ such that the total multiplicities of chains passing through any vertex $i \in L_{r}$ equals $\alpha_{r} w_{i}$.

TheOREM 6 (The Generalized LYM Property). Let $G$ be a directed acyclic graph with node weights $\mathbf{w}$, and let $L_{1}, L_{2}, \ldots, L_{s}$ be a partition of the vertices of $G$ into $s$ antichains. Let $y_{i}=w_{i} /\left\|L_{r}\right\|$, when $i \in L_{r}$. Let $w_{G}=\left(\Sigma_{r}\left\|L_{r}\right\|^{1 /(k+1)}\right)^{k+1}$. The following four conditions are equivalent:

1. $\left(k w_{G}\right) \mathbf{y}$ is an optimal solution vector to the dual convex programming problem $(D)$.

2. $\sum_{i \in A} y_{i} \leqslant 1$ for all antichains $A \in \mathbf{A}$.

3. Each $L_{r}$ is a maximal antichain and there exists a weighted regular chain covering.

4. $\sum_{i \in \Gamma(X) \cap L_{r}} y_{i} \geqslant \sum_{i \in X} y_{i}$ for any $X \subseteq L_{r}$ and any $L_{r}$ and $L_{r}^{\prime}$.

Proof. $(1) \Rightarrow(2)$ : Assume that $\left(k w_{G}\right) \mathbf{y}$ is an optimal solution vector to $(D)$, and let $\mathbf{x}$ be an optimal solution vector to $(P)$. Hence, $\max _{C \in \mathrm{C}} \sum_{i \in C} x_{i}=1$. By Theorem 2,

$$
x_{i}=\left(w_{G}^{-1} w_{i} y_{i}^{-1}\right)^{1 /(k+1)}=\left(w_{G}^{-1}\left\|L_{r}\right\|\right)^{1 /(k+1)} \text { for every } i \in L_{r} .
$$

Furthermore, the length-width equality holds for $\mathbf{x}$ and $\left(k w_{G}\right) \mathbf{y}$, in which

$$
\sum_{i \in V}\left(k w_{G}\right) x_{i} y_{i}=\sum_{r=1}^{s} \sum_{i \in L_{r}} k w_{G}^{k /(k+1)} w_{i}\left\|L_{r}\right\|^{-k /(k+1)}=\sum_{r=1}^{s} k w_{G}^{k /(k+1)}\left\|L_{r}\right\|^{1 /(k+1)}=k w_{G} .
$$

Hence, $\max _{A \in \mathbf{A}} \sum_{i \in A} y_{i}=1$.

(2) $\Rightarrow$ (1): Assume that $\max _{A \in \mathrm{A}} \sum_{i \in A} y_{i}=1$. (Note that equality holds in (2) for $A=L_{r}$, for any $r$.) Let $x$ be defined by $x_{i}=\left(w_{G}^{-1} w_{i} y_{i}^{-1}\right)^{1 /(k+1)}=\left(w_{G}^{-1}\left\|L_{r}\right\|\right)^{1 /(k+1)}$ for $i \in L_{r}$. Hence $\max _{C \in C} \sum_{i \in C} x_{i} \leqslant 1$. The length-width inequality for $\mathbf{x}$ and $\mathbf{y}$ gives $\sum_{i \in V} x_{i} y_{i} \leqslant\left(\max _{C \in \mathbf{C}} \sum_{i \in C} x_{i}\right)\left(\max _{A \in \mathbf{A}} \sum_{i \in A} y_{i}\right)=\max _{C \in \mathbf{C}} \sum_{i \in C} x_{i}$. As in the previous paragraph, we have $\sum_{i \in V} x_{i} y_{i}=1$. Hence, the length-width equality holds for $\mathbf{x}$ and $\left(k w_{G}\right) \mathbf{y}$ and by the proof of Theorem 2 they are optimal solutions.

(2) $\Rightarrow$ (3): Assume that $\max _{A \in \mathbf{A}} \sum_{i \in A} y_{i}=1$. Then every $L_{r}$ is maximal. By Dilworth's Theorem,

$$
\left.\begin{array}{ll}
\min & \sum_{C \in \mathbf{C}} \mu_{C} \\
\text { s.t. } & \mu_{C} \geqslant 0, \quad \text { each } C \in \mathbf{C}, \\
& \sum_{C: i \in C} \mu_{C} \geqslant y_{i}, \quad \text { for } i \in V .
\end{array}\right\}=\max _{A \in \mathbf{A}} \sum_{i \in A} y_{i}=1 .
$$

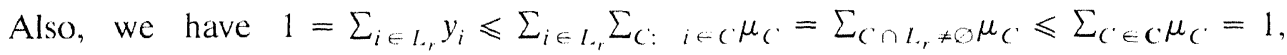
where $\left(\mu_{c}\right)$ is an optimal solution to the problem above.

Therefore, every chain $C$ with $\mu_{C} \neq 0$ intersects every $L_{r}$, and $y_{i}=\Sigma_{C: i \in C} \mu_{C}$ for every $i \in V$. The collection of chains $C$ with multiplicities $\mu_{c}$ forms a weighted regular chain covering.

(3) $\Rightarrow(4)$ : Assume that each $L_{r}$ is maximal, and there exists a weighted regular chain covering $\psi$ with multiplicities $\left\{\mu_{C}\right\}$, scaled so that $\Sigma_{C \in \psi} \mu_{C}=1$. Then it is easy 
to see from the definitions that $y_{i}=\sum_{C: i \in C} \mu_{C}$ so that

$$
\sum_{i \in X} y_{i}=\sum_{i \in X} \sum_{C: i \in C} \mu_{C}=\sum_{C: C \cap X \neq \varnothing} \mu_{C} \leqslant \sum_{C: C \cap \Gamma(X) \cap L_{r} \neq \varnothing} \mu_{C}=\sum_{i \in \Gamma(X) \cap L_{r}} y_{i}
$$

for any $X \subseteq L_{r}$. The inequality comes from the fact that $C \cap X \neq \varnothing$ implies $C \cap$ $\Gamma(X) \cap L_{r}^{\prime} \neq \varnothing$.

(4) $\Rightarrow(2)$ : Assume (4); we prove (2) by induction on the maximum number of levels met by an antichain $A$. The ground case is trivial, since $A \subseteq L_{r}$ implies $\sum_{i \in A} y_{i} \leqslant$ $\sum_{i \in L_{r}} y_{i}=1$. Assume (2) holds whenever $A$ meets less than $t$ levels and assume without loss of generality that $A$ intersects only $L_{1}, L_{2}, \ldots, L_{t}$. Let $A^{\prime}$ be the (disjoint) union of $A \cap L_{1}, A \cap L_{2}, \ldots, A \cap L_{t-1}$, and $\Gamma\left(A \cap L_{t}\right) \cap L_{t-1}$. Then $A^{\prime}$ is an antichain and $\sum_{i \in A^{\prime}} y_{i} \leqslant 1$ by induction hypothesis. But

$$
\sum_{i \in \Gamma^{M}\left(A \cap L_{\imath}\right) \cap L_{t-1}} y_{i} \geqslant \sum_{i \in A \cap L_{i}} y_{i} \text { and hence } \sum_{i \in A} y_{i} \leqslant 1 \text { as desired. }
$$

There is a further generalization of Theorem 6 to perfect graphs without requiring an anticlique (i.e., antichain) partition; see the accompanying paper by [We]. There, simultaneous "regular" coverings by cliques and anticliques are considered.

Theorems 5 and 6 can be used to obtain closed-form solutions when the conditions are satisfied. For example, the planar grid graphs, which consists of integer lattice points in the rectangle $[0, a] \times[0, b]$ with $(i, j) \rightarrow(i+1, j)$ and $(i, j) \rightarrow(i, j+1)$ (when within the specified range) satisfy the LYM property. So do the hypercubes and many other classes of graphs. (See [GK].)

6. Extension to perfect graphs. In this section, we show how the results of this paper can be extended to resource allocation problems on perfect graphs. We first need some definitions.

Consider an undirected graph $G=(V, E)$. A clique is a subset of vertices every pair of which is joined by an edge. An anticlique is a subset of vertices every pair of which is not joined by an edge. For any $W \subseteq V$, the subgraph $G_{W}$ of $G$ induced by $W$ has vertex set $W$, with an edge between two vertices in $G_{W}$ exactly when there is one in $G$. We let $\omega(G)$ and $\alpha(G)$ be the maximum number of vertices in a clique and anticlique, respectively of $G$. We let $\gamma(G)$ and $\theta(G)$ be the minimum number of anticliques and cliques, respectively, required to cover all vertices of $G$.

A graph $G=(V, E)$ is called perfect if it satisfies the following equivalent conditions:

[i] $\omega\left(G_{W}\right)=\gamma\left(G_{W}\right)$ for all $W \subseteq V$,

[ii] $\alpha\left(G_{W}\right)=\theta\left(G_{W}\right)$ for all $W \subseteq V$,

[iii] $\omega\left(G_{W}\right) \alpha\left(G_{W}\right) \geqslant|W|$ for all $W \subseteq V$.

The equivalence of these conditions is known as the Perfect Graph Theorem [Lo1, Lo2]. (See [BC, Go] for more details on perfect graphs.)

We claim that, by letting $\mathbf{C}$ and $\mathbf{A}$ be the set of all cliques and anticliques, respectively, all results of this paper remain valid for a perfect graph $G$. First, we note that the transitive closure of a directed acyclic graph gives a comparability graph which is perfect with chains corresponding to cliques, and antichains corresponding to anticliques (see [Go, Chapter 5]). So we have clearly generalized the problem statement.

The Equivalence Lemma of $\S 1$ did not use any properties of the sets $\mathbf{C}$ and $\mathbf{A}$. The Strong Duality Theorem used Lagrangian duality for convex programs and the length-width inequality and Dilworth's Theorem for directed acyclic graphs. Fulkerson 
[Fu] proved the following equivalent characterizations of perfect graphs which generalize the length-width inequality and Dilworth's Theorem:

(i) $\left(\max _{C \in \mathrm{C}} \sum_{i \in C} a_{i}\right)\left(\max _{A \in \mathbf{A}} \sum_{i \in A} b_{i}\right) \geqslant \sum_{i \in V} a_{i} b_{i}$ for all $\mathbf{a}, \mathbf{b} \geqslant \mathbf{0}$,

(ii) $\max _{A \in \mathrm{A}} \sum_{i \in A} a_{i}=\min \left\{\sum_{C \in \mathrm{C}} \mu_{C} \mid \sum_{C: i \in C} \mu_{C} \geqslant a_{i}\right.$, for all $i \in V, \mu_{C} \geqslant 0$ for all $C \in \mathbf{C}\}$ for all $\mathbf{a} \geqslant \mathbf{0}$.

Hence, the Strong Duality Theorem holds for perfect graphs.

The algorithmic results of $\S 3$ and 4 rely on the solution of the separation problem for $(P)$ in polynomial time. This is done for perfect graphs in [GLS1]. Therefore, the results of these sections carry over as well.

The Generalized LYM Property of $\$ 5$ also holds for perfect graphs where "cliques" and "anticliques" replace "chains" and "antichains".

We note that "cliques" and "anticliques" play a symmetric role in our entire development. Since the complement of a perfect graph is itself perfect, we could switch the notions of "cliques" and "anticliques" to obtain a completely parallel development. For example, the Generalized LYM Property of $\S 6$ holds true for clique partitions and regular anticlique covers.

Acknowledgements. We are very grateful to the associate editor and the referees for their comments and suggestions; in particular, the network reformulations $\left(P^{\prime}\right)$ and $\left(D^{\prime}\right)$ in $\S 2$ are due to them.

\section{References}

[BC] Berge, C. and Chvátal, V. (Editors) (1984). Topics on Perfect Graphs. Ann. Discrete Math. 21.

[BGT] Bland, R. G., Goldfarb, D. and Todd, M. J. (1981). The Ellipsoid Method: A Survey. Oper. Res. $291039-1091$.

[Di] Dilworth, R. P. (1950). A Decomposition Theorem for Partially Ordered Sets. Ann. of Math. 51 $161-166$.

[Fu] Fulkerson, D. R. (1974). Blocking and Anti-Blocking Pairs of Polyhedra. Math. Programming 1 $168-194$.

[GJ] Garey, M. R. and Johnson. D. S. (1979). Computers and Intractability: A Guide to the Theory of NP-Completeness. W. H. Freeman, San Francisco.

[Go] Golumbic, M. C. (1980). Algorithmic Graph Theory and Perfect Graphs. Academic Press, New York.

[GK] Greene, C. and Kleitman, D. J. (1978). Proof Techniques in the Theory of Finite Sets. in G.-C. Rota (Ed.), Studies in Combinatorics, MAA Studies in Math. 17 22-79.

[GLS1] Grötschel, M., Lovász, L. and Schrijver, A. (1984). Polynomial Algorithms for Perfect Graphs. in C. Berge and V. Chvátal (Eds.), Topics and Perfect Graphs, Ann. Discrete Math. 21 325-356.

[GLS2] _ and (1986). The Ellipsoid Method and the Combinatorial Optimization. Springer-Verlag, New York.

[Le] Lehman, A. (1964). A Solution of the Shannon Switching Game. J. SIAM, 12 687-725.

[Lo1] Lovász, L. (1972). Normal Hypergraphs and the Perfect Graph Conjecture. Discrete Math. 2 $253-267$.

[Lo2] (1972). A Characterization of Perfect Graphs. J. Combin. Theory Ser. B 1395-98.

[Lo3] (1986). An Algorithmic Theory of Numbers, Graphs and Contexity. SIAM, Philadelphia.

[MSTW] Monma, C. L., Schrijver, A., Todd, M. J. and Wei, V. K. (1987). A Class of Convex Programs for Resource Allocation Parallel on Directed Acyclic Graphs. Unpublished manuscript.

[Ro1] Rockafellar, R. T. (1970). Conlex Analysis. Princeton University Press, Princeton, NJ.

[Ro2] (1984). Network Flows and Monotropic Optimization. John Wiley and Sons, New York.

[U1] Ullman, J. D. (1984). Computational Aspects of VLSI, Computer Science Press.

[We] Wei, V. K. (1988). A Connection between a Convex Programming Problem and the LYM Property on Perfect Graphs. Journal of Graph Theory 12 571-587. 